\title{
Refining the role of gated SPECT for predicting clinical response to cardiac resynchronization therapy: Are we using the right tool at the wrong time?
}

\author{
Dennis A. Calnon, MD, MASNC ${ }^{\mathrm{a}}$ \\ a Cardiac Imaging, Riverside Methodist Hospital, Ohio Health Heart and Vascular Physicians, \\ Columbus, $\mathrm{OH}$
}

Received May 18, 2019; accepted May 20, 2019

doi: $10.1007 / \mathrm{s} 12350-019-01767-\mathrm{z}$

\section{See related article, pp. 1274-1284}

The accurate prediction of clinical response to cardiac resynchronization (CRT) remains challenging, despite much work in this area. International guidelines for the use of CRT are currently based solely on the severity of heart failure despite optimal medical therapy, reduced left ventricular ejection fraction (LVEF), electrical dyssynchrony (QRS duration), and atrial rhythm. ${ }^{1}$ However, as many as $30-40 \%$ of patients who meet patient selection criteria fail to derive clinical benefit following CRT. ${ }^{2}$

It is logical to assume that patient selection for CRT would be enriched by limiting CRT to patients who demonstrate evidence of mechanical dyssynchrony at baseline. Although the presence of mechanical dyssynchrony at baseline is associated with better response rates, baseline echocardiographic measures of mechanical dyssynchrony are not sufficient for identifying patients who derive clinical benefit from CRT. ${ }^{3}$ For this reason, echocardiographic measurement of dyssynchrony is not routinely performed prior to CRT in current clinical practice.

Phase analysis of gated single-photon emission computed tomography (SPECT) myocardial perfusion imaging (MPI) is a powerful and robust technique for measuring left ventricular mechanical dyssynchrony. ${ }^{4}$

Reprint requests: Dennis A. Calnon, MD, MASNC, Cardiac Imaging, Riverside Methodist Hospital, Ohio Health Heart and Vascular Physicians, Columbus, OH , USA; Dennis.Calnon@OhioHealth.com J Nucl Cardiol 2020;27:1285-7.

$1071-3581 / \$ 34.00$

Copyright (C) 2019 American Society of Nuclear Cardiology.
Advantages of phase analysis of gated SPECT MPI include:

1. Availability (standard SPECT cameras and standard 8 frame gating).

2. Superior reproducibility and repeatability.

3. Ability to identify the site of latest mechanical activation.

4. Ability to assess for presence and extent of scar at the site of left ventricular (LV) lead placement.

5. The ability to make repeated measurements of mechanical dyssynchrony using a single radionuclide tracer injection.

Despite these theoretical advantages, phase analysis of gated SPECT has not been incorporated into clinical decision making for CRT. In this issue, Patel et al. report the effect of CRT on septal perfusion and septal thickening 6 months post implantation in 105 patients with symptomatic heart failure despite medical therapy, nonischemic cardiomyopathy with $\mathrm{LVEF} \leq 35 \%$, QRS duration $\geq 120 \mathrm{msec}$, and left bundle branch block (LBBB). ${ }^{5}$ As expected, improvement in septal thickening at 6 months correlated with an increase in LVEF, reduction of end-systolic volume (ESV), end-diastolic volume (EDV), and left ventricular dyssynchrony. There was no relationship between baseline septal thickening and the primary outcome (improvement in NYHA class, improvement in LVEF by $\geq 5 \%$, reduction of ESV by $\geq$ $15 \%$, and improvement of at least 5 points in Minnesota Living with Heart Failure Questionnaire (MLHFQ). Relative perfusion of the septal wall, anterior wall, and inferior wall increased post-CRT, with a decrease in relative tracer uptake in the lateral wall compared to baseline. The result is "normalization" to a more homogeneous myocardial perfusion pattern. As the authors acknowledged, the baseline septal perfusion 
defect on SPECT MPI might be partly related to the LBBB and the associated wall motion abnormality via the partial volume effect, as this pattern is not observed with higher resolution PET MPI in the setting of LBBB. ${ }^{6}$

The study by Patel et al. in this issue represents a subgroup analysis of the VISION-CRT multicenter trial reported by Peix et al. ${ }^{7}$ The VISION-CRT study included 195 patients from 8 countries and gated SPECT dyssynchrony assessment was performed with phase standard deviation (PSD) before and 6 months after CRT. The baseline dyssynchrony (PSD) was not associated with clinical improvement with CRT by univariable or multivariable analyses, suggesting that baseline measurement of PSD would not be useful for selecting patients who are likely to benefit from CRT. However, the change in PSD from baseline to 6 months post-CRT was significantly associated with clinical improvement. From a practical perspective, predicting clinical benefit from CRT based on measurement of PSD 6 months after lead implantation would be less useful, as the patient would have already been exposed to the costs and potential complications associated with CRT.

A more practical approach would be to assess for improvement in dyssynchrony immediately prior to and after CRT. Using echocardiographic assessment of dyssynchrony and multivariable logistic analysis, immediate resynchronization was shown to be the only variable that was predictive of response to CRT at 6 months follow-up. ${ }^{3}$ When patients showed $<20 \%$ resynchronization immediately after CRT, response to CRT never occurred at 6 months. Baseline measures of dyssynchrony were similar between CRT responders and non-responders, and therefore baseline dyssynchrony assessment was not helpful in predicting response to CRT.

Assessment of dyssynchrony by phase analysis of gated SPECT would be ideally suited for assessment of the acute response to CRT. The 6-hour half-life of the technetium-labeled SPECT tracers would permit serial measurements of dyssynchrony (PSD) immediately before and after CRT using a single radionuclide tracer injection. The measurements of PSD could be repeated in multiple LV lead positions if the coronary venous anatomy allows for more than one location suitable for lead placement. An immediate improvement in dyssynchrony (PSD) would provide confidence that the patient will derive long-term clinical benefit from CRT. If there is no immediate improvement in dyssynchrony based on measurements of PSD in multiple LV lead positions, then the patient would be very unlikely to benefit from CRT and the decision could be made to defer permanent placement of the LV lead. Compared to echocardiographic assessments of dyssynchrony, the advantages of the phase analysis gated SPECT approach would be the superior reproducibility and repeatability of the PSD measurements, as well as the ability to identify the site of latest mechanical activation and the presence of myocardial scar. The disadvantage of using gated SPECT to assess immediate response to CRT would be the logistical challenge of securing access to an available SPECT camera in relatively close proximity to the electrophysiology laboratory.

In order for phase analysis of gated SPECT MPI to be incorporated into clinical guidelines for selecting patients for CRT, a multicenter clinical trial would be needed to confirm the feasibility and predictive accuracy of dyssynchrony assessment with PSD immediately prior to and after LV lead placement. For purposes of the clinical trial, the CRT would be performed according to current clinical guidelines with permanent implantation of the LV lead even if immediate assessment by PSD demonstrates no improvement in dyssynchrony compared to baseline. The predictive accuracy of PSD measured immediately post-CRT would be determined by measuring clinical and echocardiographic response at 6 months.

Phase analysis of gated SPECT MPI is a powerful and robust tool for assessment of dyssynchrony, but it is currently left unused in our nuclear cardiology toolbox. Perhaps if we begin to measure PSD immediately before and after CRT, we will finally put this powerful tool to use.

\section{Disclosure}

Dennis A. Calnon have no financial conflicts to disclose.

\section{References}

1. Normand C, Linde C, Singh J, Dickstein K. Indications for cardiac resynchronization therapy: A comparison of the major international guidelines. J Am Coll Cardiol HF 2018;6:308-16.

2. Abraham WT, Fisher WG, Smith AL, et al. Cardiac resynchronization in chronic heart failure. N Engl J Med 2002;346:1845-53.

3. Bleeker G, Mollema SA, Holman ER, et al. Left ventricular resynchronization is mandatory for response to cardiac resynchronization therapy: Analysis in patients with echocardiographic evidence of left ventricular dyssynchrony at baseline. Circulation 2007;116:1440-8.

4. Chen J, Garcia EV, Bax JJ, et al. SPECT myocardial perfusion imaging for the assessment of left ventricular mechanical dyssynchrony. J Nucl Cardiol 2011;18:685-94.

5. Patel C, Kalaivani M, Karthikeyan G, et al. Effect of cardiac resynchronization therapy on septal perfusion and septal thickening: Association with left ventricular function, reverse remodeling, and dyssynchrony. J Nucl Cardiol 2019. https://doi.org/10.1007/s12350019-01704-0.

6. Nowak B, Stellbrink C, Schaefer WM, et al. Comparison of regional myocardial blood flow and perfusion in dilated 
cardiomyopathy and left bundle branch block: Role of wall thickening. J Nucl Med 2004;45:414-8.

7. Peix A, Karthikeyan G, Massardo T, et al. Value of intraventricular dyssynchrony assessment by gated-SPECT myocardial perfusion imaging in the management of heart failure patients undergoing cardiac resynchronization theraoy (VISION-CRT). J Nucl Cardiol 2019. https://doi.org/10.1007/s12350-018-01589-5.

Publisher's Note Springer Nature remains neutral with regard to jurisdictional claims in published maps and institutional affiliations. 